EPJ Web of Conferences 41, 01010 (2013)

DOI: $10.1051 /$ epjconf/20134101010

(C) Owned by the authors, published by EDP Sciences, 2013

\title{
The Simplest Method for Generation of an Attosecond Pulse Train
}

\author{
Kazumichi Yoshii, John Kiran Anthony, and Masayuki Katsuragawa \\ Department of Engineering Science, University of Electro-Communications, 1-5-1 Chofugaoka, \\ Chofu-shi, Tokyo, Japan
}

\begin{abstract}
We report an extremely simple approach to generate an attosecond pulse train from more than octave-spanning discrete spectrum by only positioning transparent materials into the optical path without spatially dispersing the frequency components.
\end{abstract}

\section{Introduction}

The field of attoscience [1] such as the attosecond metrology [2] and the electronic dynamics in atoms and molecules [3] has developed in parallel with the first achievement of an attosecond pulse generation with high-order harmonic generation [4,5]. This development has encouraged intensive study on the generation of few-femtosecond to attosecond pulses by synthesizing frequencies over more than octave bandwidth [6,7]. Recently, an octave-spanning discrete spectrum produced from molecular modulator has received much attention as a new source for few-cycle pulse train and arbitrary waveform generation [8-11].

In this work, we report a new and extremely simple approach to generate an attosecond pulse train from such an octave-spanning discrete spectrum by only positioning optical glass plates or crystals into the optical path without spatially dispersing the frequency components. For any broad bandwidth, this approach can almost perfectly produce the transform limited (TL) pulse. We demonstrate the generation of attosecond pulse train with the pulse duration of 730 as and the period of the pulse-train of 8.03 fs. The present method is robust and applicable to high power laser source.

\section{Numerical experiment}

In order to produce TL pulses, we only need to find the condition by adding positive dispersion, where the set of spectral phases realize a linear relationship. Interestingly, such condition can be found in a large number within a realistic thickness of dispersive medium. Moreover, the search for such condition is equal to solving numerical optimization problem with few parameters, and therefore we can easily obtain the conditions by using existing numerical calculations.

We perform a numerical experiment consisted of generating a discrete spectrum with a bandwidth of more than $1 \mathrm{PHz}$ and compressing the pulse train down to attosecond regime by using this method. Figure 1 shows a schematic diagram assumed as the actual experimental setup. We calculate a coupled propagation equation for vibrational-Raman-coherence in parahydrogen with the conditions shown in Figure 1, and gain the amplitudes and the spectral phases for 12 Raman sidebands. Furthermore, we consider that the sidebands are transmitted through a window and a lens made of

This is an Open Access article distributed under the terms of the Creative Commons Attribution License 2.0, which permits unrestricted use, distribution, and reproduction in any medium, provided the original work is properly cited. 
fused silica with the total thickness of $50 \mathrm{~mm}$. The maximum points for revival peak intensity of the intensity waveforms are searched with fused silica, sapphire (ordinary ray), and $\mathrm{CaF}_{2}$ as dispersive media.

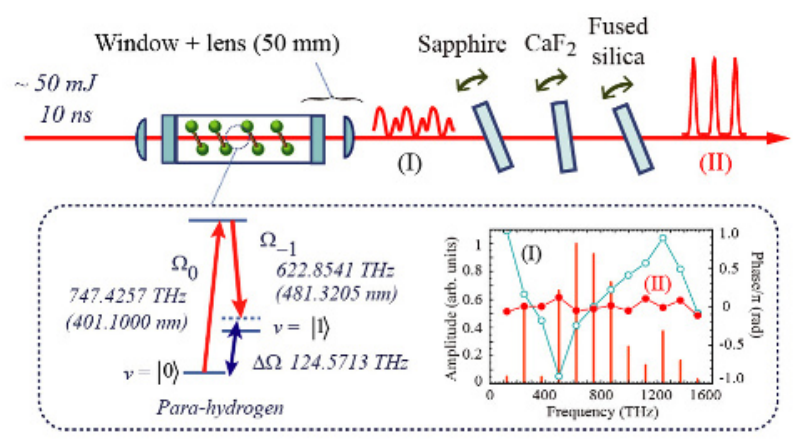

Fig. 1. Schematic diagram of numerical experiment for generating an attosecond pulse train. Two driving-fields $\Omega_{-1}=622.8541 \mathrm{THz}(481.3205 \mathrm{~nm})$ and $\Omega_{0}=747.4257 \mathrm{THz}(401.1000$ $\mathrm{nm})$ adiabatically drive a high vibrational-Raman-coherence in parahydrogen $\left(5.38 \times 10^{19}\right.$ $\mathrm{cm}^{-3}$ ), where the pulse duration is $10 \mathrm{~ns}$ and the peak excitation-intensity is $5.0 \mathrm{GW} / \mathrm{cm}^{2}$. The difference frequency $\Delta \Omega=124.5713 \mathrm{THz}$ is detuned by $-300 \mathrm{MHz}$ from the exact Ramanresonance $(v=0 \rightarrow 1)$. The 12 Raman sidebands are generated. The sidebands are transmitted through a window and a lens made of fused silica, where total thickness is $50 \mathrm{~mm}$. Dispersive media are fused silica, sapphire (ordinary ray), and $\mathrm{CaF}_{2}$. The power spectrum and spectral phases are shown in the inset.

\section{Results and discussion}

We obtained many points where the peak intensity recovers more than $90 \%$ of the original peak intensity. In Figure 2(a), the peak intensity variations as functions of the added thicknesses of dispersive media are shown. The maximum peak intensity is 0.963 at $x_{\mathrm{FS}}=7.387 \mathrm{~mm}, x_{\mathrm{Sa}}=4.878$ $\mathrm{mm}$, and $x_{\mathrm{CaF} 2}=1.786 \mathrm{~mm}$. The inset shows an enlarged view around the maximum point. The points corresponding to more than $90 \%$ of the original peak intensity are distributed in an oblong shape with considerable volume. Therefore, the search for the maximum point can be easily done by controlling the thicknesses of dispersive media with the practical resolution.

Figure 2(b) shows the temporal intensity waveforms corresponding to the spectral phases at the point (I) and (II) in Figure 1. The spectral phases of dispersive media are calculated from Sellmeier equations. The revival pulse duration is 730 as at full width at half maximum (FWHM), which almost matched the Fourier-transform-limited duration of 728 as and the period of the pulse train is 8.03 fs. This result shows that the high power attosecond pulse train can be generated by using just three dispersive media. We also confirmed that this method can be applied to frequency components with any initial spectral phases.

The present method is closely related to the so called "self-imaging phenomenon" [12,13], where the spectral phases of revival pulse are controlled to be equal to integer multiples of $\pi$. This method is, however, absolutely different from the self-imaging method. The usual self-imaging method can never function for such a broad spectrum over an octave. 
(a)

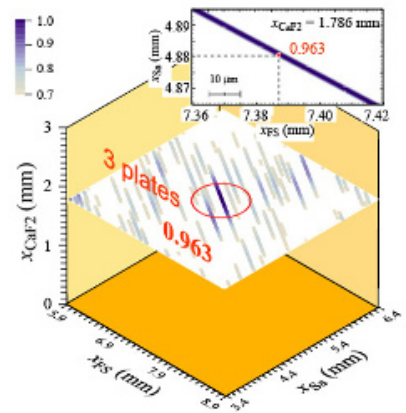

(b)

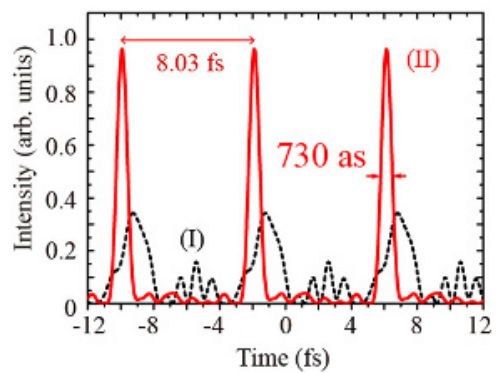

Fig. 2. (a) Normalized peak intensity variation of the intensity waveforms as functions of the dispersive media. The inset shows enlarged view around maximum peak. Maximum value is 0.963 at $x_{\mathrm{FS}}=7.387 \mathrm{~mm}, x_{\mathrm{Sa}}=4.878 \mathrm{~mm}$, and $x_{\mathrm{CaF} 2}=1.786 \mathrm{~mm}$. (b) Temporal intensity waveforms reconstructed with the spectral phases in (I) (dashed line) and (II) (solid line). The revival pulse duration is 730 as at FWHM (TL: 728 as) and the period of the pulse-train is $8.03 \mathrm{fs}$.

\section{Summary}

We have shown the novel method to generate an attosecond pulse train from a broad discrete spectrum by simply placing a few transparent media in the optical path and adjusting their effective thicknesses. The method was numerically applied to a broad discrete spectrum with a bandwidth of 1.4 PHz generated thorugh the molucular modulation technique, and the 730-attosecond pulse train was successfully produced. The present method is extremely simple and robust, and also applicable to high power lasers.

\section{References}

1. P. B. Corkum, F. Krausz, Nature Physics 3, 381 (2007)

2. M. Hentschel, R. Kienberger, Ch. Spielmann, et al., Nature 414, 509 (2001)

3. G. Sansone, F. Kelkensberg, J. F. P. Torres, et al., Nature 465, 763 (2010)

4. R. Kienberger, E. Goulielmakis, M. Uiberacker, et al., Nature 427, 817 (2004)

5. G. Sansone, E. Benedetti, F. Calegari, et al., Science 314, 443 (2006)

6. R. K. Shelton, L. S. Ma, H. C. Kapteyn, et al., Science 293, 1286 (2001)

7. A. Wirth, M. Th. Hassan, I. Grguraš, et al., Science 334, 195 (2011)

8. S. E. Harris and A. V. Sokolov, Phys. Rev. Lett. 81, 2894 (1998)

9. T. Suzuki, M. Hirai, and M. Katsuragawa, Phys. Rev. Lett. 101(24), 243602 (2008)

10. H. S. Chan, Z. M. Hsieh, W. H. Liang, et al., Science 331, 1165 (2011)

11. S. Baker, I. A. Walmsley, J. W. G. Tisch, and J. P. Marangos, Nature Photonics 5, 664 (2011)

12. J. Azana, and M. A. Muriel, IEEE J. Sel. Top. Quantum Electron. 7(4), 728 (2001)

13. T. Suzuki, and M. Katsuragawa, Opt. Express 18, 23088 (2010) 